\title{
Polio Eradication in Bangladesh: Evaluation of AFP Surveillance Indicators, 2011-2015
}

\author{
Khan Redzwan Habib \\ World Health Organisation, Dhaka, Bangladesh
}

Email address:

khan.redz@gmail.com,habibk@who.int

\section{To cite this article:}

Khan Redzwan Habib. Polio Eradication in Bangladesh: Evaluation of AFP Surveillance Indicators, 2011-2015. International Journal of Immunology. Vol. 5, No. 1, 2017, pp. 11-19. doi: 10.11648/j.iji.20170501.13

Received: January 8, 2017; Accepted: January 17, 2017; Published: February 20, 2017

\begin{abstract}
As per recommendation from WHO, Bangladesh began a comprehensive plan for AFP (Acute Flaccid Paralysis) and EPI (Expanded Program on Immunisation) disease surveillance from 1997. The country has been maintaining key performance indicators for AFP surveillance for the past ten years. Bangladesh has been declared polio free on $27^{\text {th }}$ March 2014. The aim of this paper is to evaluate and describe results of AFP surveillance indicators for Bangladesh showing the progress made over time as well as identifying areas, which needs further improvements to maintain the polio free status. This retrospective study has been conducted using the surveillance data routinely collected from AFP cases on demographic characteristics and clinical \& laboratory examination. Data of 7480 children less than 15 years old reported between January 2011 to December 2015 have been included in this study. Among all reported cases 59.22\% cases were male children with a male: female ratio of 1.45:1. 54\% of the reported AFP cases within the study period belong to 1-4 years of age. Among all reported cases, $56 \%$ have been diagnosed with Guillain-Barre Syndrome or GBS. Annual Non-Polio AFP rate has been successfully achieved nationally for each study year. Completeness and Timeliness of passive reporting from facilities have been satisfactory. $98 \%$ or more of all reported AFP cases within the study period has been investigated within 48 hours of notification. For over $95 \%$ of all reported AFP cases two stool samples were collected within 14 days of case reporting. 60 plus day follow up has been performed for at least $99 \%$ reported AFP cases within the study period. All epidemiological and laboratory surveillance indicators were successfully met for AFP surveillance. However it is important for Bangladesh to maintain long-term AFP case based surveillance along with introduction of Environmental surveillance due to the risk of poliovirus importation before global polio eradication.
\end{abstract}

Keywords: AFP Surveillance, Surveillance Indicators, Polio Eradication, Bangladesh

\section{Introduction}

During World Health assembly held in May 1988, ministers of all WHO member countries voted to launch a global program to eradicate poliomyelitis. As a result of this initiative the global polio eradication program started. Rapidly the incidence of polio cases decreased by $99 \%$ globally and soon three WHO regions (Americans, Western pacific and European) were certified polio-free [1]. In WHO South-East Asian Region (SEAR) intensive polio eradication program using tOPV also has lead to substantial decrease in number of polio cases and eleven countries of this region have been declared polio free on $27^{\text {th }}$ March 2014 [2].

Bangladesh, one of the eleven member countries of WHO South-East Asian Region (SEAR), is the eight most populated country in the world and fifth most populated in Asia [3]. The country is a role model for the world for its achievements in immunisation program, which started as Expanded Program on Immunisation or EPI in 1979. The program has prevented an estimated two million child deaths from 1987-2000 and continues to do so each year [4]. The country has achieved an impressive $92.3 \%$ crude fully vaccinated rate by 2014 from a crude fully vaccinated rate of $76 \%$ in 1995 [5].

Before 1986 an estimated 11,500 children used to develop polio every year in Bangladesh [6]. As a part of the global program to polio eradication the country has conducted its first National Immunisation Day (NID) on March 16 and April 16 of the year 1995 [7]. Bangladesh had zero reported polio cases since 2000 up to 2006 when the country became 
re-infected with poliovirus imported into the country. Tests suspected the virus to be transmitted from Uttar Pradesh district of neighbouring country India. After six months of extensive operations, six rounds of special immunisation days (SIDs) and mop-up campaigns once again polio has been eradicated from the country by November 2006. The country has been polio free since and has been certified as a polio free country with ten other WHO SEAR regions in 2014. The country till 2014 has observed 21 NIDs for polio among which the last one held in January 2014 reached a confirmed coverage of $100 \%$ [8].

Data about reported cases of poliomyelitis in Bangladesh has been available since 1988. Vigorous testing for poliovirus from stool began in 1992 and national Acute Flaccid Paralysis (AFP) case reporting began in 1996. As per recommendation from WHO, Bangladesh began a comprehensive plan for AFP and EPI disease surveillance from 1997, which includes development of facility, training of health professionals and community based surveillance system [9]. Since poliovirus is not the only cause of acute flaccid paralysis, a broad definition including other diseases, which also cause AFP like symptoms among children 15 years or below, has been introduced for AFP surveillance. To maximise and evaluate the surveillance activity, certain performance indicators have also been introduced [10]. Bangladesh has achieved all performance indicators for AFP surveillance and has been sustaining this achievement for past ten years [8].

Bangladesh though enjoying the polio free status, the country needs constant on going surveillance activities as in two of its neighbouring countries Pakistan and Afghanistan polio still remains endemic. In 2011 China reported polio outbreak after a gap of ten years, which was found to be related to wild poliovirus circulating in Pakistan [11]. Thus if a polio free country becomes re-infected as Bangladesh and China did in the past, this could spread like wild fire. Bangladesh following the Global Polio Eradication Initiative's "Polio Eradication and Endgame Strategic Plan" [12] has successfully made the switch from tOPV to IPV and has withdrawn tOPV from its immunisation program.

With the country's switch from tOPV to IPV it is the right time to look back and evaluate the AFP surveillance activities to keep the system and healthcare staffs updated and identify bottleneck situation if there is any. Therefore, the aim of this study is to evaluate and describe results of AFP surveillance indicators for Bangladesh showing the progress made over time as well as identifying areas, which needs further improvements to maintain the polio free status.

\section{Method}

\subsection{Study Design}

Bangladesh has seven administrative divisions with sixtyfour districts. Total population of the country as of 2016 is $162,903,941$. Approximately $34.3 \%$ population is under 15 years of age [13]. Immunisation in Bangladesh is performed through fixed and outreach sites with the help of health assistants (HA) and at times family welfare assistants (FWAs) to assist them [14]. At present EPI in the country provides vaccination against number of fatal diseases under routine immunisation schedule. These are: childhood Tuberculosis, Diphtheria, Pertussis, Tetanus, Hepatitis B, Hemophilus Inuenza type B, Poliomyelitis, Measles, Rubella and Pneumococcal Pneumonia [5]. Government of Bangladesh funds $56 \%$ of total spending in the vaccination program [15].

A retrospective study has been conducted using the surveillance data routinely collected by Institute of Epidemiology, Disease Control and Research (IEDCR) and World Health Organisation-Bangladesh, from January 2011 to December 2015. Immediately after case notification two stool samples are collected and sent to WHO accredited National Polio \& Measles Laboratory (NPML) for investigation. All AFP cases reported to disease surveillance department of IEDCR and NPML within the mentioned period have been included in this study. WHO has set certain performance indicators for AFP surveillance [16, 17]. This study has discussed and compared these performance indicators to evaluate the surveillance activity in the country.

\subsection{Surveillance Activity}

Immunisation and Vaccine Development (IVD) program of WHO-Bangladesh has been providing technical assistance to Government of Bangladesh through its network of Surveillance Medical Officers (SMO) in strengthening the immunisation system including vaccine preventable diseases and adverse events following immunisation (AEFI) surveillance [18].

The SMOs along with other immunisation activities ensures that all AFP cases be notified and investigated as prospective polio cases immediately maintaining timeliness and completeness as advised by WHO. When a suspected AFP case is reported to the local health facility, SMOs with assistance from local health authorities investigate the patient according to the AFP case definition. A case investigation form is containing demographic information, clinical history and vaccination history of the reported AFP case filled up. SMOs also ensure collection of two stool samples 24 hours apart within 14 days of onset of paralysis through government staffs and the investigation form along with the stool sample is sent to NPML for further laboratory investigation. SMOs ensure that copies of this investigation form are preserved in local WHO office, in district EPI headquarters \& also in upazilla level health centres (UHC). NPML after performing laboratory investigation of the stool samples sends back the investigation form along with laboratory investigation reports. Once again the SMO network make sure that the previous copies of the investigation form from all health facilities are replaced with the investigation form and laboratory report sent by NPML. SMOs also conduct a 60-plus day follow up of all reported AFP cases to rule out signs of residual weakness. 


\subsection{Laboratory Investigation and Final Classification of AFP Cases}

WHO accredited National Polio \& Measles Laboratory (NPML) in Dhaka is equipped to isolate poliovirus from stool sample, identify poliovirus to confirm wild variety and differentiate the three poliovirus serotypes. NPML follows four standard timeliness indicators: (i) $\geq 80 \%$ stool sample reaches the lab within 72 hours of collection; (ii) $\geq 90 \%$ stool sample reaches the lab in good condition; (iii) $\geq 80 \%$ stool sample results are complete within 14 days and (iv) isolation of $\geq 10 \%$ non-polio enterovirus (NPEV) from stool samples.

AFP cases for which the mentioned criteria is not met or residual paralysis remains after 60 days of paralysis onset or for which patients dies or gets lost in follow up are considered to be ERC cases. ERC or Expert Review Committee is a national body of experts who after going through the medical and demographic history of the cases for which stool sample could not be collected or was inadequate, give the final decision whether to consider the case as a compatible polio case or not.

\subsection{Data Analysis}

Demographic data was entered in Microsoft Excel 2011 and frequencies and tables were generated. To compare percentages, Pearson Chi-square test was performed using SPSS and a $p$ value $<0.005$ has been considered significant.

\subsection{Ethical Approval}

The study has received ethical approval from Ministry of Health and Family Welfare (MOHFW) and from Institute of Epidemiology, Disease Control and Research (IEDCR).

\section{Result}

Expected annual non-polio AFP cases to be reported within 2011-2015 were 5427, which has been calculated using the total number of under 15 years of age children in the country in these five years (expected annual non-polio AFP rate in Bangladesh is $\geq 2 / 100,000$ less than 15 years of age population). A total number of 7480 AFP cases under the age of 15 years have been reported within the study period. Among all the reported cases by the time this study has been conducted, 12 were pending classification and the rest 7468 cases have been discarded. There were no compatible cases and no case with isolation of wild poliovirus has been diagnosed within the study period (Table: 1 ).

Table 1. Summary of AFP Cases in Bangladesh, 2011 - 2015.

\begin{tabular}{lllll}
\hline & $\mathbf{2 0 1 1}$ & $\mathbf{2 0 1 2}$ & $\mathbf{2 0 1 3}$ & $\mathbf{2 0 1 4}$ \\
\hline Expected annual non-polio AFP cases & 1075 & 1085 & 1099 & 1112 \\
Total reported AFP cases & 1610 & 1567 & 1412 & 1478 \\
Number of discarded cases & 1610 & 1567 & 1412 & 1478 \\
Number of compatible cases & 0 & 0 & 0 & 0 \\
Number of cases with isolation of wild poliovirus & 0 & 0 & 0 & 0 \\
Number of cases pending classification & 0 & 0 & 0 & 0 \\
\hline
\end{tabular}

*From 2005 Non-Polio AFP cases have been calculated considering 2 cases per 100,000 under 15 population

**Data as of 19 March 2016

Among all reported cases $59.22 \%$ cases were male and $40.78 \%$ were female children with a male: female ratio of $1.45: 1.54 \%$ of the reported AFP cases within the study period belong to $1-4$ years of age (Figure: 1 ).

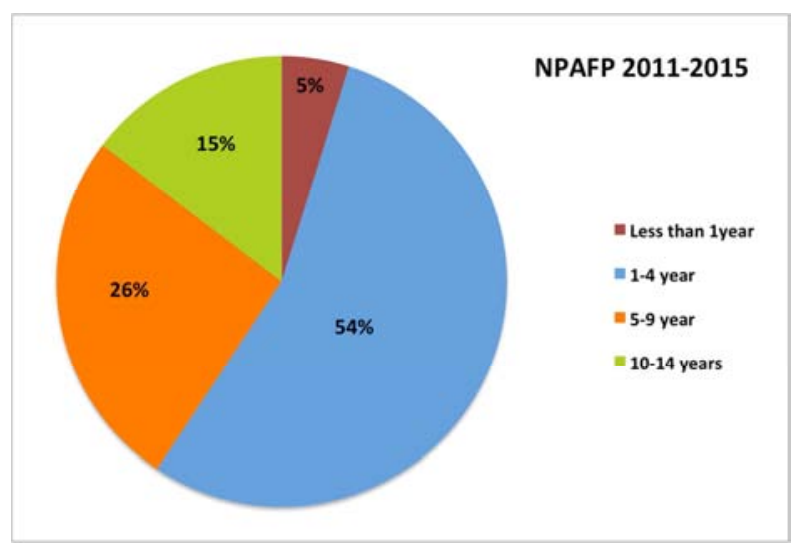

Figure 1. Age Distribution of NPAFP Cases (2011-2015).

A noticeable increases in case reporting has been observed from May to August of each year with number of AFP cases decreasing gradually as the years comes to an end (Figure: 2 ).

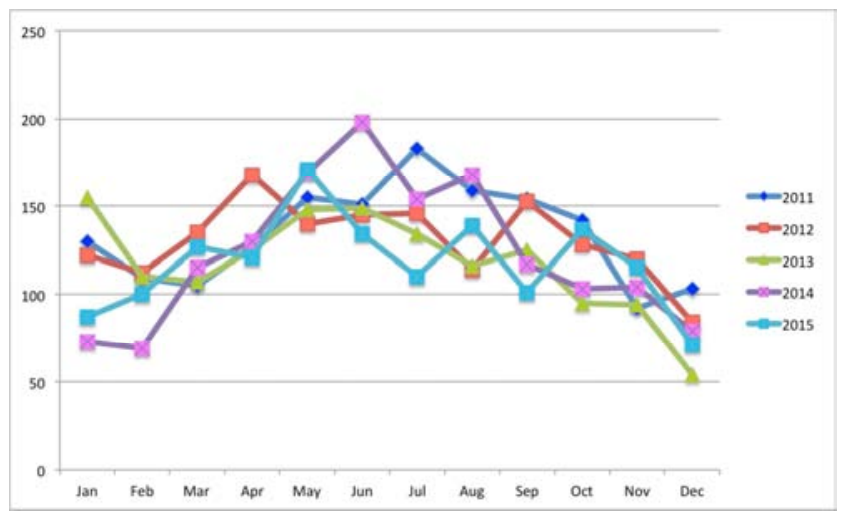

Figure 2. Comparison of AFP Cases Reported by Month of Onset (20112015).

Vaccination status has been satisfactory of all reported AFP cases, as for each year over $90 \%$ of all reported cases have received 4-7 doses of OPV. Numbers of reported AFP cases who have received not even a single dose of OPV or for whom vaccination status have been unknown are very low (Figure: 3). 


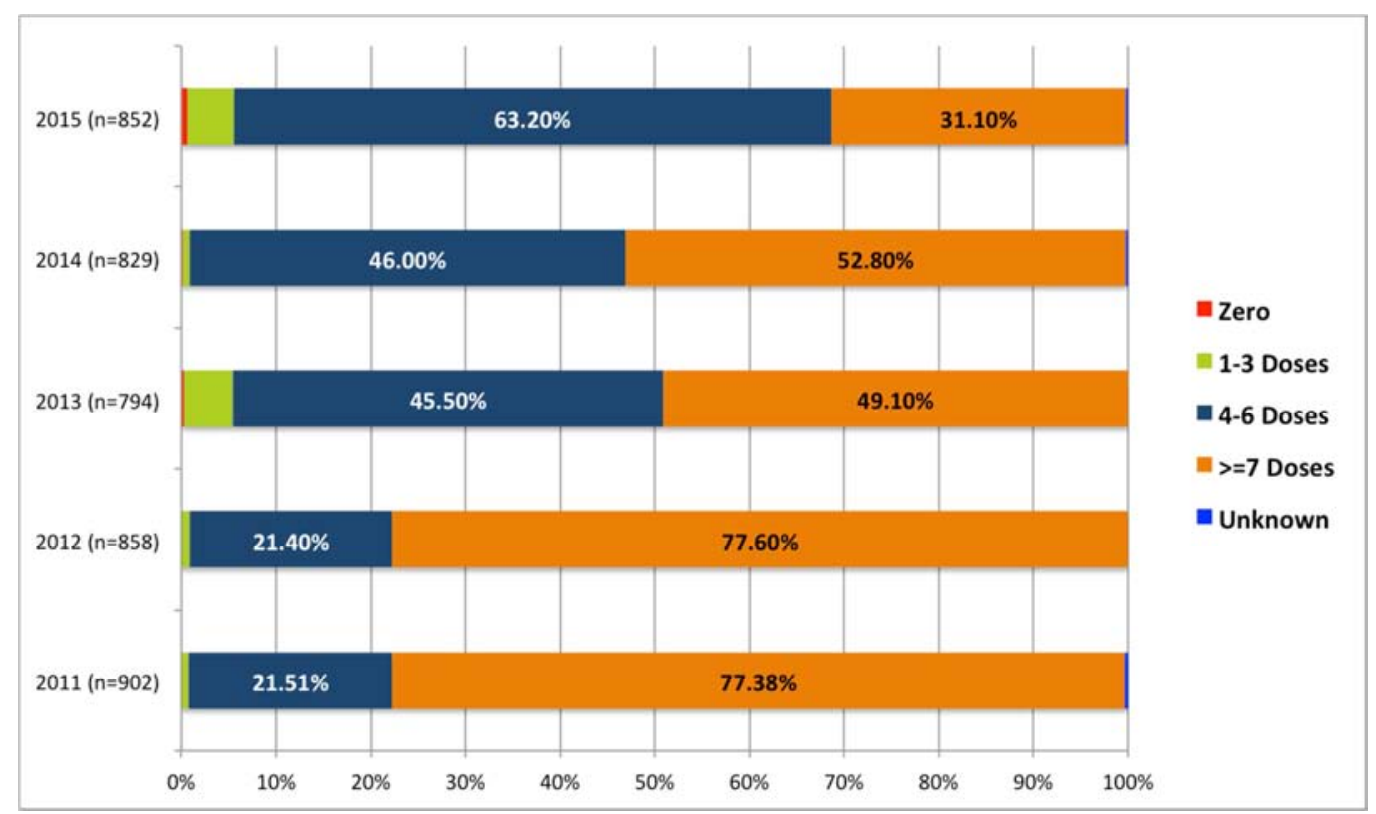

Figure 3. OPV status (Routine+NIDs) of 6-59 months NPAFP Cases, (2011-2015).

Among all reported AFP cases within the study period, $56 \%$ have been diagnosed with Guillain-Barre Syndrome or GBS. The next highest is Myositis and others with both gaining $14 \%$ of all reported AFP cases (Figure: 4 ).

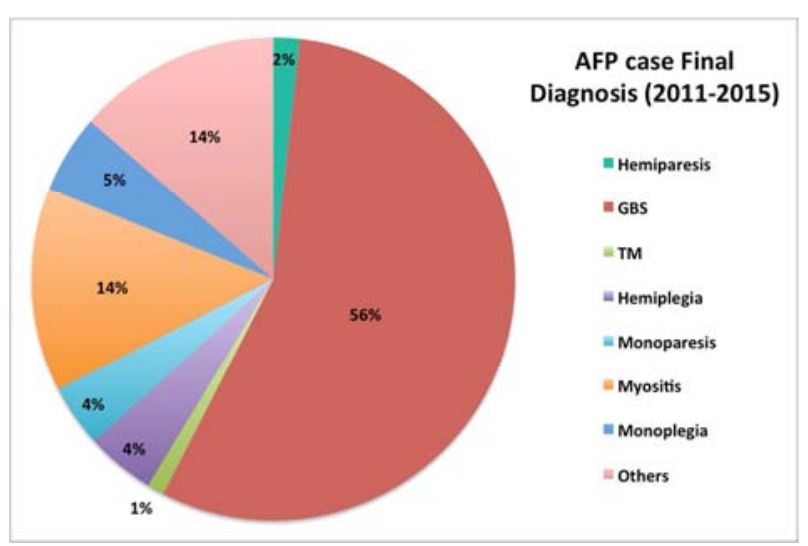

Figure 4. Final diagnosis of reported AFP cases (2011-2015).
Table 2 shows the overall evaluation of AFP surveillance performance indicators in Bangladesh from 2011-2015. All performance indicators are successfully met within this time period. The most important performance indicator 'Annual Non-Polio AFP rate' which is the indicator of sensitivity of the surveillance system, has been successfully achieved nationally for each study year. Also Completeness and Timeliness of passive reporting from facilities that is when reports of AFP cases are sent by designated facilities or designated individuals from these facilities, have also been satisfactory. Both these indicators are found to be gradually improving with time reaching $97 \%$ for completeness and $92 \%$ for timeliness in the year 2015. Indicator for stool samples reaching the national affiliated laboratory (NPML) for laboratory investigation within 3 days of collection has been $100 \%$ for each study year except for 2013 even when it was $99 \%$.

Table 2. AFP Surveillance Performance Indicators Bangladesh 2011-2015.

\begin{tabular}{|c|c|c|c|c|c|c|}
\hline & Target & 2011 & 2012 & 2013 & 2014 & 2015 \\
\hline Annual Non-Polio AFP rate & $>2.0$ & 3.11 & 2.98 & 2.65 & 2.74 & 2.75 \\
\hline Completeness of passive reporting from facilities & $>90 \%$ & $92 \%$ & $91 \%$ & $91 \%$ & $96 \%$ & $97 \%$ \\
\hline Timeliness of passive reporting from facilities & $>80 \%$ & $86 \%$ & $85 \%$ & $85 \%$ & $89 \%$ & $92 \%$ \\
\hline AFP cases investigated within 48 hours of notification & $>80 \%$ & $99 \%$ & $98 \%$ & $99 \%$ & $99 \%$ & $98 \%$ \\
\hline AFP cases with 2 stool samples within 14 days & $>80 \%$ & $95 \%$ & $96 \%$ & $96 \%$ & $98 \%$ & $97 \%$ \\
\hline Stool samples at Lab within 3 days of collection & $>80 \%$ & $100 \%$ & $100 \%$ & $99 \%$ & $100 \%$ & $100 \%$ \\
\hline Stool samples at Lab in good condition & $>90 \%$ & $100 \%$ & $100 \%$ & $100 \%$ & $100 \%$ & $100 \%$ \\
\hline Stool samples with Lab results & $>80 \%$ & $91 \%$ & $84 \%$ & $96 \%$ & $95 \%$ & $96 \%$ \\
\hline Isolation of NPEV from stool samples & $>10 \%$ & $18 \%$ & $14 \%$ & $19 \%$ & $23 \%$ & $20 \%$ \\
\hline
\end{tabular}

All seven divisions achieved $>2$ Annual Non-Polio AFP rate within the study period except for Sylhet division barely missing the target in 2013 (Figure: 5). The highest number of AFP cases has been reported in 2011 with the Annual Non-Polio AFP rate reaching to 3.11. Among seven divisions of the country Barisal division is found to report the highest number of AFP cases consistently. 


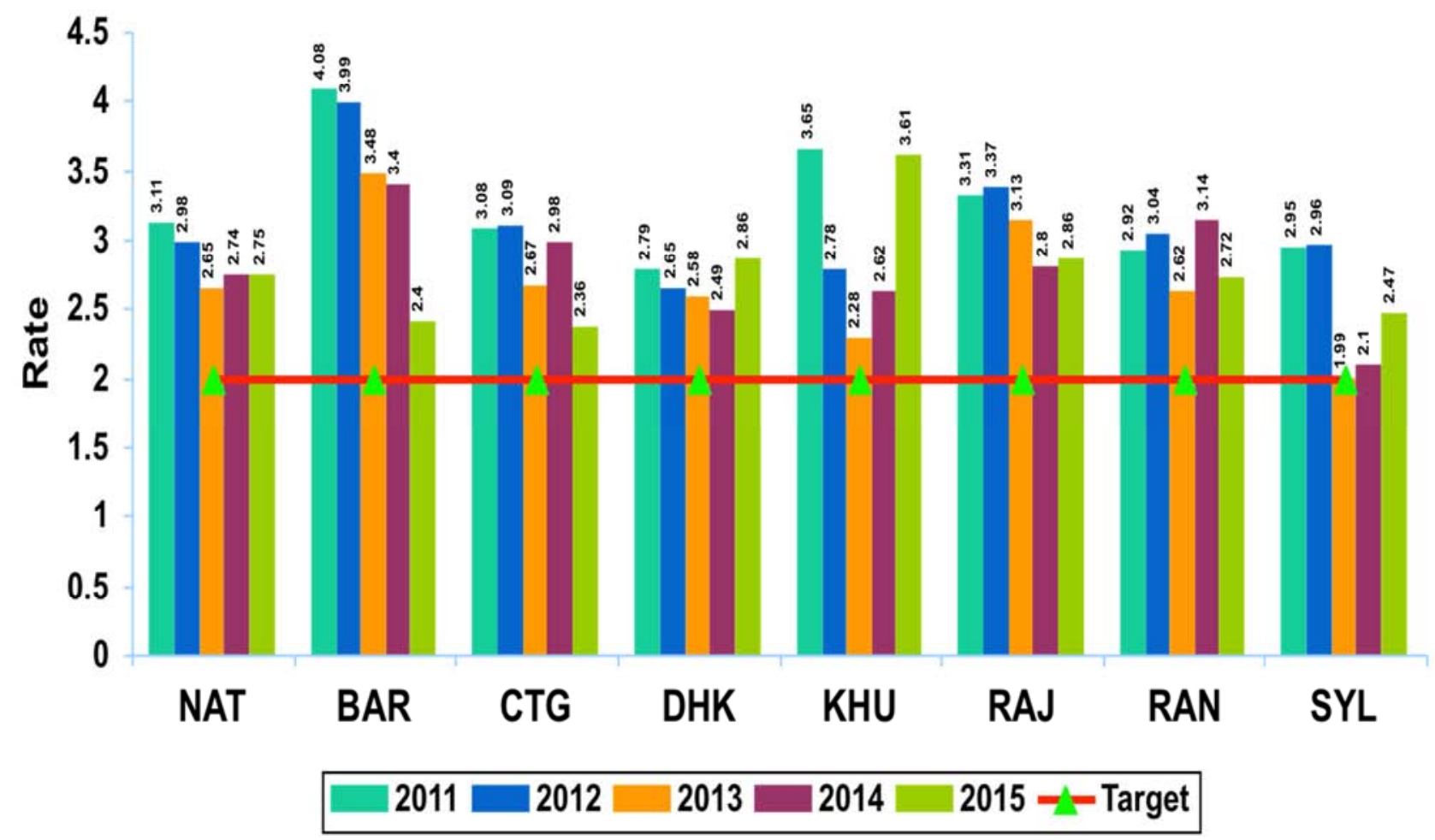

NAT $=$ National; BAR $=$ Barisal CTG $=$ Chittagong; DHK $=$ Dhaka; KHU $=$ Khulna; RAJ $=$ Rajshahi; RAN $=$ Rangpur; $\mathrm{SYL}=\mathrm{Sylhet}$.

Figure 5. Annuslised Non-Polio AFP rate by division (2011-2015).

98\% or more of all reported AFP cases within the study period has been investigated within 48 hours of notification (Figure: 6). In the years 2011, 2013 and 2014 an impressive 99\% of all reported AFP cases has been investigated within 48 hours of notification.

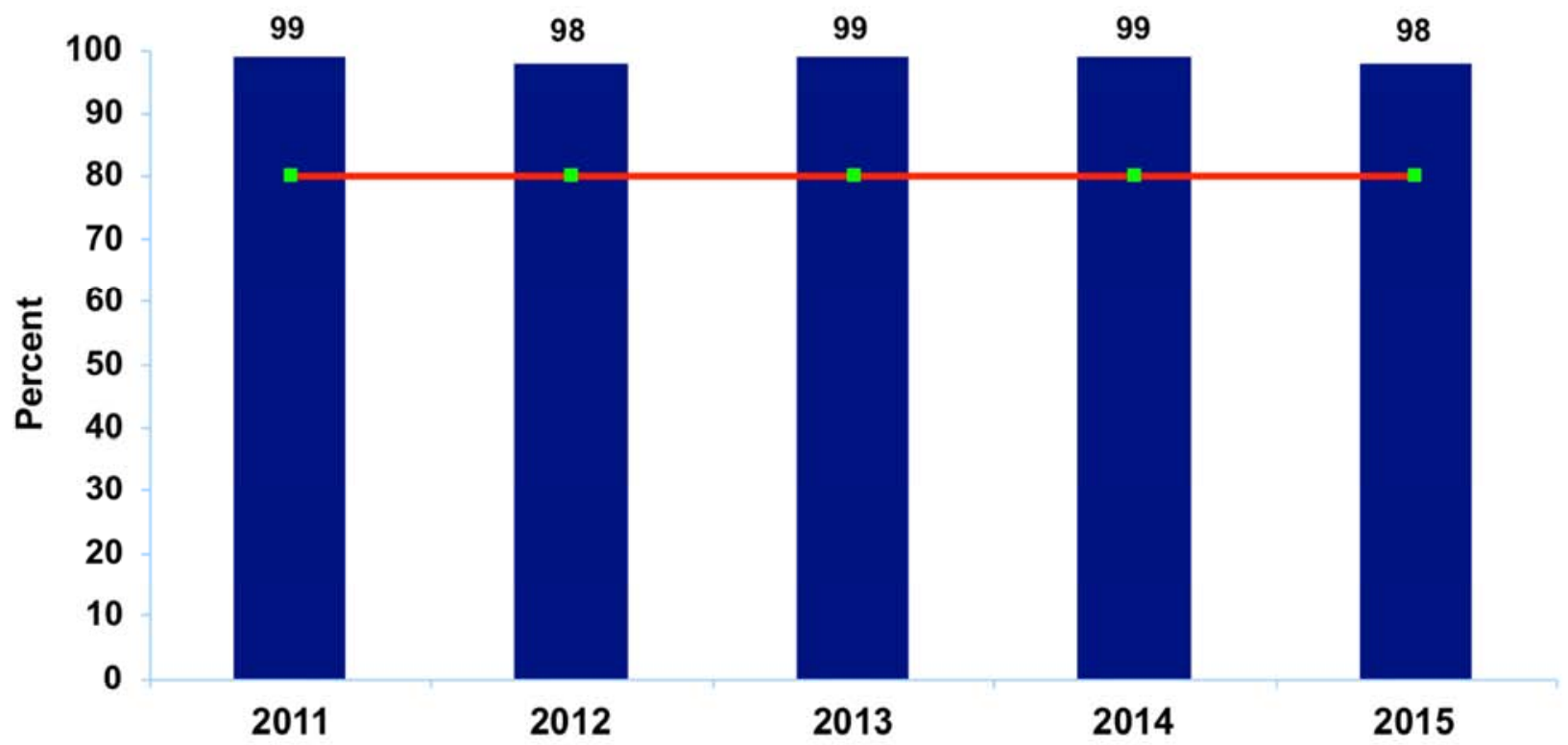

Figure 6. Rate of AFP Cases Investigated within 48 Hours of Notification, (2011-2015).

For over $95 \%$ of all reported AFP cases two stool samples were collected within 14 days of case reporting (Figure: 7). This surveillance indicator like others has also maintained a gradual improvement with time. 


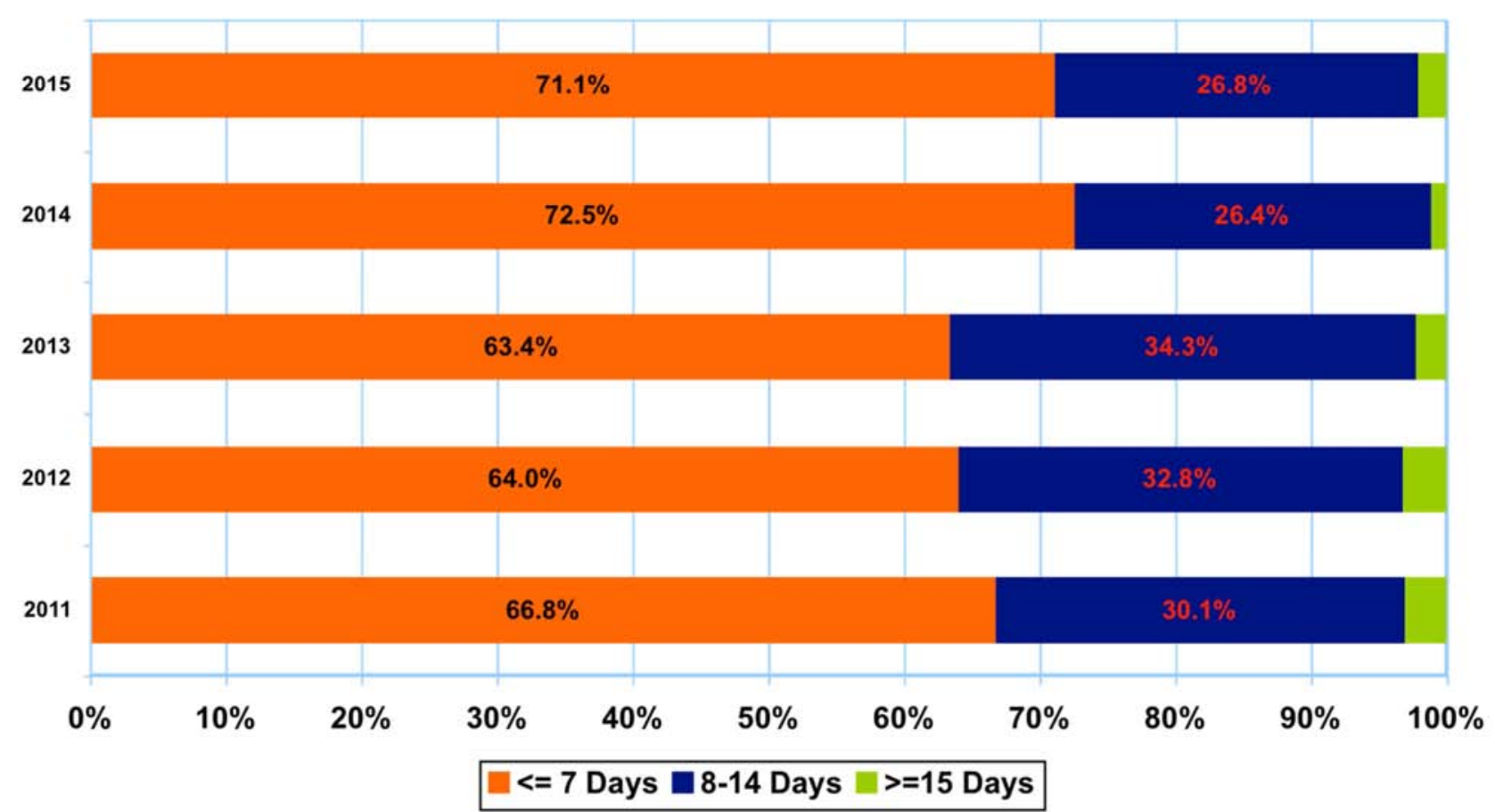

Figure 7. AFP cases with 2 stool samples within 14 days, (2011-2015).

60 plus day follow up has been performed for 100\% reported AFP cases in 2011, 2013 and 2015. The highest number of AFP cases for which a 60 plus day follow up has been conducted were 399 in 2011 (Figure: 8). Over $80 \%$ of all 60 plus day follow up have been conducted within 30 days.

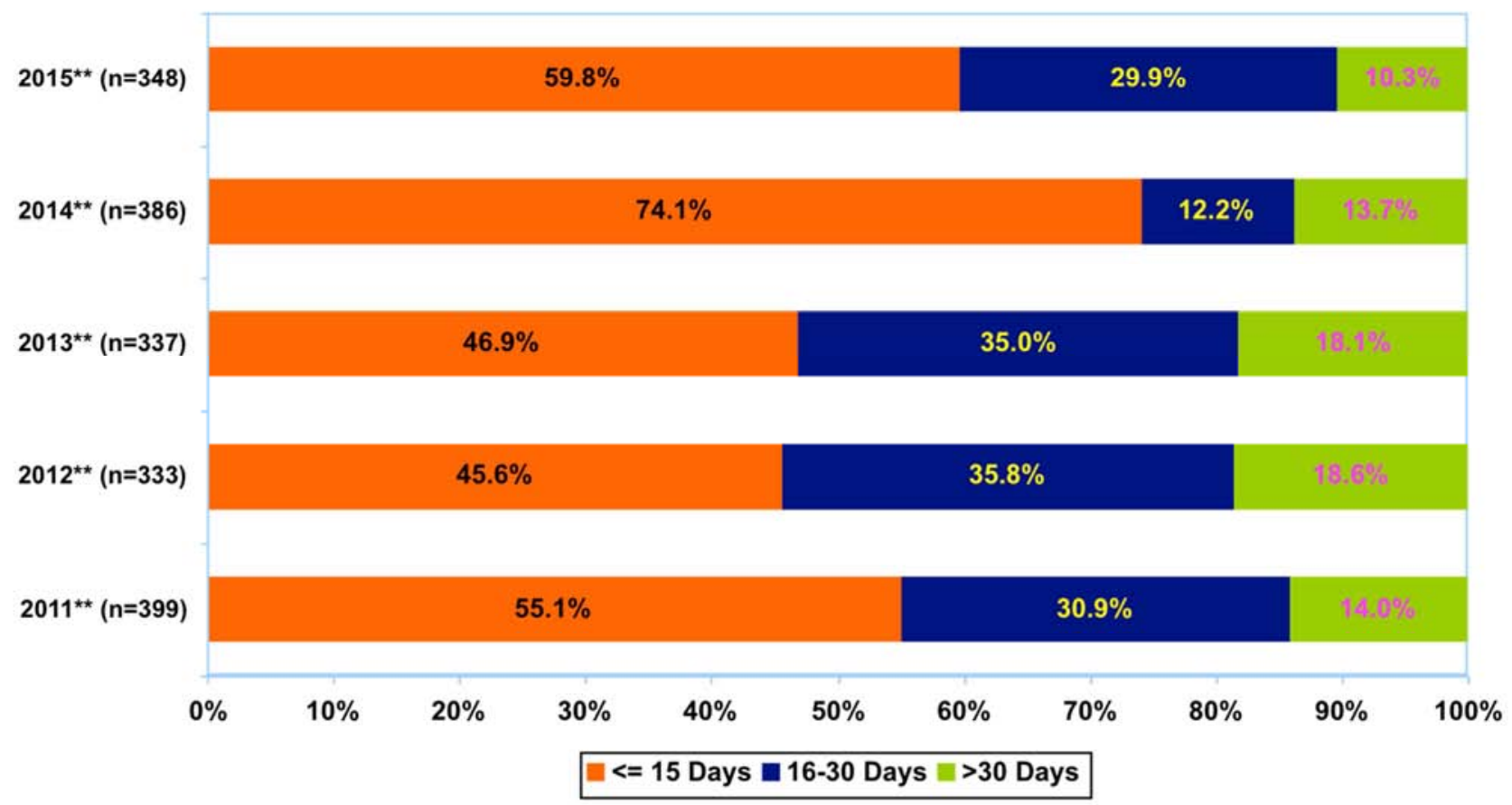

Figure 8. Comparison of 60+day follow-up exam of AFP Cases (2011-2015).

** Analysis was done of the cases having inadequate stool, positive isolates at NPL \& NPEV and cases are not included in the denominator where follow-up result comes as Died or Lost to Follow-up

A national average of 23 AFP cases for which isolation of Non-polio enterovirus from stool samples have been done were in 2014, which is also the highest within the study period. Among the divisions Rangpur fell short in 2012 only by two cases. 
Barisal division once again is found to perform well with highest 31 cases in 2014 from which isolation of non-polio enterovirus have been done (Figure: 9).

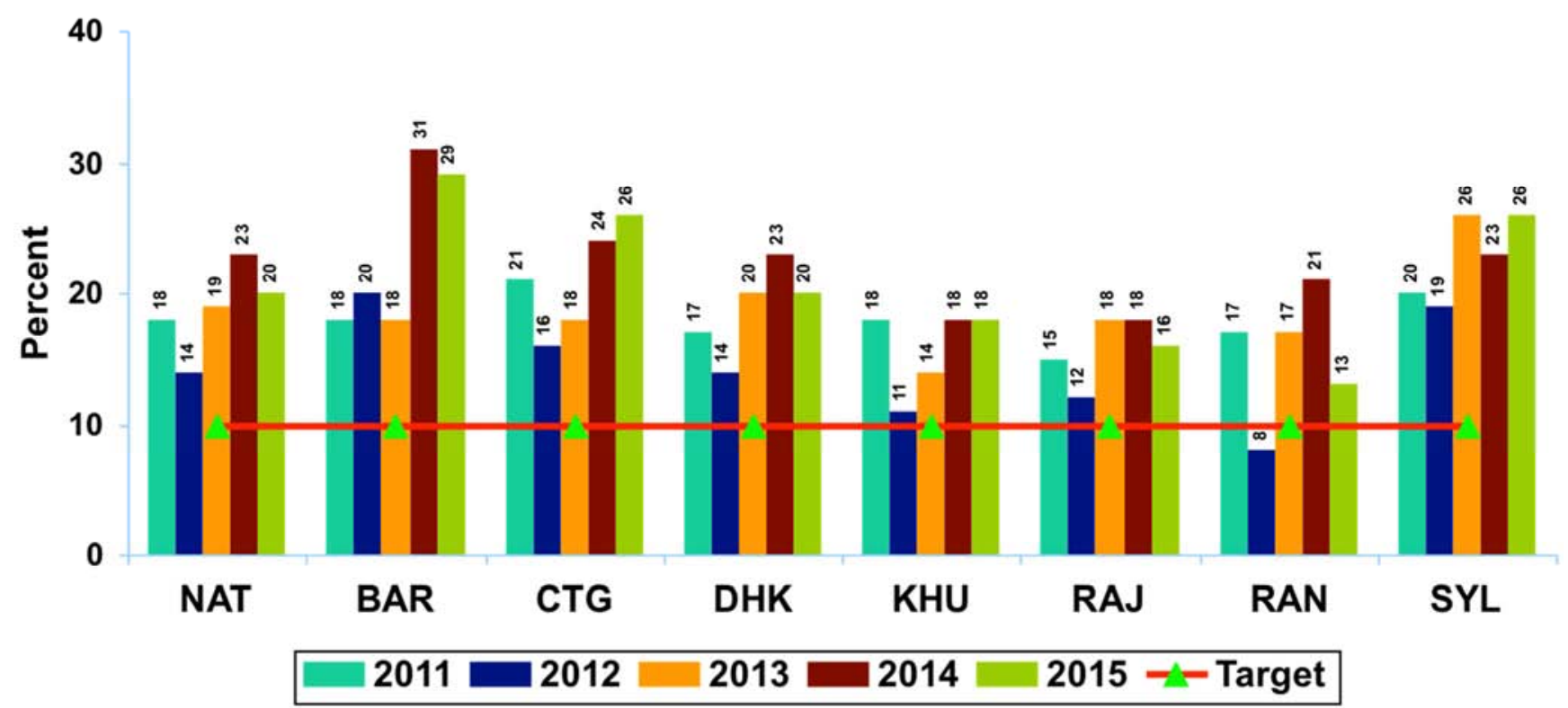

NAT $=$ National; BAR $=$ Barisal CTG $=$ Chittagong; DHK $=$ Dhaka; KHU $=$ Khulna; RAJ $=$ Rajshahi; RAN $=$ Rangpur; $\mathrm{SYL}=\mathrm{Sylhet}$.

Figure 9. Isolation of NPEV from stool samples.

\section{Discussion}

This study represents a comprehensive national surveillance status of Bangladesh and reports the evaluation of the Acute Flaccid Paralysis surveillance performance indicators from 2011 to 2015. It is note worthy to mention that the country has reported 36 compatible cases in 2001 and 2 compatible cases each in 2004 and 2006. In 2006 the country has reported 18 wild poliovirus cases. However from 2007 till this study period the country has reported neither any wild poliovirus nor any compatible case. Though it was only from 2005, the target of non-polio AFP rate was set to 2/100,000 under 15 years age children [19] but from 2001 till 2015 annualised non-polio AFP rate has always been well over 2 in the country.

The study has identified that $59 \%$ of total reported AFP cases are below the age of 5 years (59 months). This finding is consistent with a study conducted in Ireland, a country of WHO EURO region where $51.4 \%$ of total reported AFP cases were below the age of 5 years [20]. But this percentage of age distribution is different than in India $(90 \%)$ another country within the WHO SEAR region [21]. For data available of Marches region of Italy another country of WHO EURO region $37 \%$ of total reported AFP cases belong to under 5 years [22]. For Ibadan, the state capital of Oyo state in Nigeria a country belonging to WHO AFRO region $74.4 \%$ of total reported AFP cases belong to under 5 years children [23].

This study has found higher incidence of AFP among boys than in girls, $59.22 \%$ and $40.78 \%$ respectively though it is not statistically significant $(p=0.992)$. The similar findings of higher incidence of AFP among boys than in girls were observed in other studies as well $[22,24]$.

More polio cases are being reported at the middle of the year usually as found in this study. Specially reporting starts to increase from May and this trend continues till August. From this point onwards case reporting tends to decrease with the lowest number of AFP cases being reported in November and December. This finding is similar to a study conducted in India [25] but is different than in Ireland where more AFP cases are reported (34.3\%) in the autumn that is from August till October [20].

For all reported AFP cases vaccination status have been found satisfactory in this study. In 2011/12 more than $75 \%$ of all reported AFP cases were found to receive 7 or more doses of OPV but in later years of the study period that is 20132015 this trend changed. Approximately half of all AFP cases reported in 2013-2015 have received 4-6 doses of OPV while approximately the rest half have received more than seven doses. Overall well over $90 \%$ of all reported AFP cases were found to receive 4-7 or more doses of OPV. The country was able to achieve this impressive coverage due to repetitive successful NIDs.

Along with satisfactory achievements in the OPV coverage, this study has found Bangladesh constantly performing well in AFP surveillance performance indicators. 98-99\% of all reported AFP cases within this study period have been investigated within 48 hours of notification. For $95 \%$ of all AFP cases two stool samples have been collected within 14 days. For well over $60 \%$ cases two stool samples were collected within less than 7 days of case notification. Additionally data analysis has indicated that all the divisions in the country were able to meet this performance indicator despite of their distance from NPML in Dhaka. The study has 
identified that in $95 \%$ occasions or more stool samples were being collected from reported AFP cases and for 99\% times within this study period these stool samples have reached NPML with 72 hours of collection. Data also indicates that collected stool samples were adequate in amount and had reached NPML in good condition. All seven divisions in the country were able to meet this criterion.

60 plus day follow up has constantly been performed for almost $100 \%$ cases where it were necessary. All seven divisions have been performing well to isolate non-polio enterovirus (NPEV) from stool samples within the study period. One important finding of the study was that none of the 64 districts of the country have gone silent within the study period. On few occasions though few upazillas having 100,000 or more below 15-year-old children were silent. Therefore, it is important to keep all upazillas equally sensitised of AFP case reporting as incase of possible poliovirus transmission and circulation it could go undetected.

\section{Conclusion}

Considering all finding and analysis of AFP surveillance performance indicators this study has come to a conclusion that AFP surveillance activities have been satisfactory in Bangladesh from 2011-2015. The credit for this will go to strong surveillance network of World Health Organisation in the country and also to maximum sensitivity of field staffs about AFP case reporting. In addition to maintain this best practice, this study suggests the country taking the next step to contribute in global polio eradication. As mentioned earlier in this study, Bangladesh has successfully made the switch from tOPV to IPV and has withdrawn tOPV from its immunisation program following the "Polio Eradication and Endgame Strategic Plan" [12]. Researches suggest that Environmental Poliovirus Surveillance (ENV) should be in place during the period between interruption of wild poliovirus (WPV) transmission \& certification of eradication and should be continued into the post eradication phase and OPV cessation period to monitor emergence of Vaccine Derived Poliovirus (VDPV) re-emergence of WPV or disappearance of all OPV-related strains [26]. There is also evidence that ENV along with routine AFP case based surveillance is more effective to detect introduction and circulation of Wild Poliovirus (WPV) and Vaccine Derived Poliovirus (VDPV) [27-31]. Environmental surveillance also has a role in new WHO strategy for intensified effort to complete poliomyelitis eradication [32] and can be useful to monitor the efficacy of immunisation initiatives when necessary [26].

Several industrial countries like USA, UK, Australia, Germany, Canada have successfully made the switch from OPV to IPV in the past and no signs of emerging transmission of OPV related viruses have been detected in those countries [26]. However, concern remains whether the same will be true for developing countries with high population like Bangladesh. Furthermore, two neighbouring countries of Bangladesh - Pakistan and Afghanistan where polio still remains endemic might put the country's polio free status at risk.

Therefore, considering all possibilities it will be the right time to introduce and expand Environmental surveillance in the country to increase the sensitivity of overall poliovirus detection. This study strongly suggests that the country keeps continuing it's ongoing excellent AFP case based surveillance activity along with introduction of effective Environmental surveillance system to maintain the polio-free status.

\section{References}

[1] Sutter, R. W., et al., Poliomyelitis eradication: progress, challenges for the end game, and preparation for the posteradication era. Infectious Disease Clinics of North America, 2001. 15 (1): p. 41-64.

[2] World Health Organisation. WHO South-East Asia Region is officially certified polio-free. 2014; Available from: http://www.searo.who.int/entity/campaigns/poliocertification/en/.

[3] Global Alliance for Vaccines and Immunisation, GAVI Country Factsheet: Bangladesh. 2016, Global Alliance for Vaccines and Immunisation.

[4] Global Alliance for Vaccines and Immunisation, Government of People's Republic of Bangladesh May 2011 Comprehensive Multi-Year Plan. 2011.

[5] Directorate General of Health Services Bangladesh. Bangladesh EPI Coverage Evaluation Survey 2014. 2014 May 11, 2016]; Available from:

http://www.dghs.gov.bd/images/docs/EPI/EPIEvaluationSurve y2014.pdf.

[6] UNICEF. 21st NID to reinforce country's polio-free status. 2013 [cited 2016 June 16]; Available from: http://www.unicef.org/bangladesh/media_8593.htm.

[7] Bhattacharyya, K. and R. Khanam, Process Evaluation of the First National Immunization Day in Bangladesh. 1998.

[8] World Health Organisation, Bangladesh: home-grown solutions helped stop polio and keep this country polio-free. 2014, World Health Organisation: Bangladesh.

[9] CDC, Progress Toward Poliomyelitis Eradication -Bangladesh, 1995-1997. 1998, Centre for Disease Control: Wasington DC.

[10] World Health Organisation, Polio Laboratory Manual. 2004, World Health Organisation: Geneva, Switzerland,

[11] Chatterjee, A., S. Vidyant, and T. N. Dhole, Polio eradication in India: Progress, but environmental surveillance and vigilance still needed. Vaccine, 2013. 31 (9): p. 1268-1275.

[12] World Health Organisation, Polio Eradication \& Endgame Strategic Plan 2013-2018. 2013, World Health Organisation: France.

[13] Global Alliance for Vaccines and Immunisation, Bangladesh. 2016, GAVI. 
[14] Habib, K. R., Evaluating Immunisation Dropout Rates in Eight Hard to Reach Unions of Moulvibazar District, Bangladesh 2016.

[15] World Health Organisation, EPI Fact Sheet: Bangladesh 2014. 2014, World Health Organisation.

[16] World Health Organisation Fiield Guide: For Supplementary Activities aimed at achieving polioeradication-1996 revision. 1997.

[17] CDC Updated guidelines for evaluating public health surveillance systems. Recommendations from the Guidelines Working Group. 2001.

[18] World health Organisation, Immunization and Vaccine Development. 2016, World Health Organisation-Bangladesh: Bangladesh.

[19] CDC, Progress Toward Interruption of Wild Poliovirus Transmission --- Worldwide, January 2005--March 2006. 2005: Washington, DC.

[20] Flanagan, P., et al., Reporting of acute flaccid paralysis in children under 15 years of age: improving surveillance, January 2009-December 2014. Irish Medical Journal, 2016.

[21] Singh, K., G. Kaur, and R. Kumar, Acute paralytic poliomyelitis: change in number over years impact of PPI: sentinel centre experience. Indian Journal of Community Medicine, 2004. 29 (2): p. 82.

[22] D'Errico, M. M., et al., Surveillance of acute flaccid paralysis in the Marches region (Italy): 1997-2007. BMC infectious diseases, 2008. 8 (1): p. 1 .

[23] Hamzat, T. K. and T. T. Omotade, Acute flaccid paralysis: a five-year review of cases managed by physiotherapy at the University College Hospital, Ibadan. African journal of health sciences, 2006. 13 (1): p. 28-32.
[24] Odoom, J. K., et al., Evaluation of AFP surveillance indicators in polio-free Ghana, 2009-2013. BMC public health, 2014. 14 (1): p. 1.

[25] Hanumaiah, H., et al., Non-polio Enteroviruses in Karnataka, India: Virological surveillance of acute flaccid paralysis cases (July 1997-2013). Indian journal of medical microbiology, 2016. 34 (1): p. 22.

[26] Hovi, T., et al., Role of environmental poliovirus surveillance in global polio eradication and beyond. Epidemiology and infection, 2012. 140 (1): p. 1.

[27] Pöyry, T., M. Stenvik, and T. Hovi, Viruses in sewage waters during and after a poliomyelitis outbreak and subsequent nationwide oral poliovirus vaccination campaign in Finland. Applied and environmental microbiology, 1988. 54 (2): p. 371-374.

[28] Van der Avoort, H., et al., Isolation of epidemic poliovirus from sewage during the 1992-3 type 3 outbreak in The Netherlands. Epidemiology and infection, 1995. 114 (03): p. 481-491.

[29] Dedepsidis, E., et al., Retrospective characterization of a vaccine-derived poliovirus type 1 isolate from sewage in Greece. Applied and environmental microbiology, 2007. 73 (21): p. 6697-6704.

[30] Shulman, L. M., et al., Neurovirulent vaccine-derived polioviruses in sewage from highly immune populations. PLoS One, 2006. 1 (1): p. e69.

[31] Asghar, H., et al., Environmental surveillance for polioviruses in the Global Polio Eradication Initiative. Journal of Infectious Diseases, 2014. 210 (suppl 1): p. S294-S303.

[32] World Health Organisation, The Global Polio Eradication Strategic Plan, 2010-2012. 2010: Geneva, Switzerland. 